\title{
Nuclear Magnetic Resonance Study of the Intramolecular Nonmutual Exchange of 5-Halobenzofuroxan. I. Application of Density Matrix Method ${ }^{11}$
}

\author{
Shogo Uematsu and Yukio AKahori
}

Shizuoka College of Pharmacy?2)

(Received April 25, 1977)

\begin{abstract}
The tautomerism of 5-fluorobenzofuroxan was studied by the proton magnetic resonance at 60 and $100 \mathrm{MHz}$. The rate of intramolecular rearrangement between 5-fluorobenzofuroxan $(\mathrm{ABCX})$ and 6-fluorobenzofuroxan $\left(\mathrm{A}^{\prime} \mathrm{B}^{\prime} \mathrm{C}^{\prime} \mathrm{X}^{\prime}\right)$ was obtained by analyzing the line shapes of proton magnetic resonance signals. The theoretical spectra were calculated by means of a computer program based on the density matrix theory of the nonmutual exchange developed by Johnson, and the theoretical spectra showed a good agreement with the experimental line shapes in the temperature range from $-78^{\circ}$ to $+30^{\circ}$ Activation parameters for the intramolecular rearrangement were found to be $\Delta H^{\ddagger}=5 \sim 10$ $\mathrm{kcal} / \mathrm{mol}$ and $\Delta S^{*}=-33 \sim-17 \mathrm{cal} / \mathrm{deg} \cdot \mathrm{mol}$.
\end{abstract}

Keywords-NMR; density matrix method; tautomerism; rearrangement; benzofuroxan; 5-fluorobenzofuroxan

\section{Introduction}

Boulton and Ghosh ${ }^{3)}$ stated that benzofuroxans are heterocyclic compounds and exist in two isomeric forms which undergo rapid intramolecular rearrangement in solution at a moderate temperature. Tautomerism of benzofuroxans has mainly been studied by nuclear magnetic resonance (NMR) spectral analysis and a stable form was found to be the 6 -isomer for nitro derivatives $\left(\mathrm{R}=\mathrm{NO}_{2}\right)$, and the 5 -isomer for chloro derivatives $\left.(\mathrm{R}=\mathrm{Cl}),{ }^{4}\right)$ and the free energy of activation is about $15 \mathrm{kcal} / \mathrm{mol}$ for intramolecular rearrangement.

In the present-day, it was recognized that the most reasonable mechanistic pathway for this tautomerism involves a ring opening of the furazan oxide to give $o$-dinitrosobenzene as a transient intermediate to afford either $\mathrm{Ia}$ or $\mathrm{Ib}$ (Chart 1 ).

Using bond energy arguments, Mallory and Cammarate ${ }^{5)}$ showed that other possibilities are far less likely but no sound evidence has appeared for the presence of intermediary

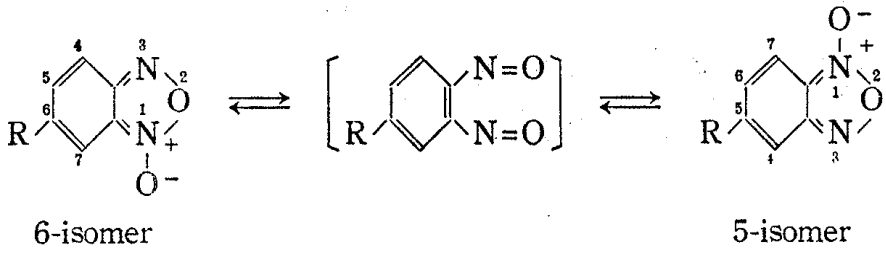

Chart 1

1) A part of this work was presented at the 93rd Annual Meeting of the Pharmaceutical Society of Japan, Tokyo, April, 1973, and 95th Annual Meeting of the Pharmaceutical Society of Japan, Nishinomiya, April, 1975.

2) Location: 2-2-1, Oshika, Shizuoka, 422, Japan.

3) A.J. Boulton and P.B. Ghosh, Advan. Heterocycl. Chem., 10, 1 (1969).

4) R.K. Harris, A.R. Katritzky, S. Oksne, A.S. Bailey, and W.G. Paterson, J. Chem. Soc., 1963, 197; A.J. Boulton, A.R. Katritzky, M.J. Sewell, and B. Wallis, J. Chem. Soc. B, 1967, 914.

5) F.B. Mallory, S.L. Manatt, and S. Wood, J. Am. Chem. Soc., 87, 5433 (1965); F.B. Mallory and A. Cammarate, ibid., 88, 61 (1966). 
o-dinitrosobenzene. The present kinetic study was undertaken to provide more precise thermodynamic constants by an application of the density matrix method to the intramolecular nonmutual exchange reaction.

A quantum mechanical treatment, based on the density matrix formalism, was first presented by Karplan ${ }^{6)}$ and further developed by Alexander. ${ }^{7)}$ Karplan and Alexander's theory is limited to the case involving a mutual exchange of nuclear spins (benzofuroxan $(\mathrm{R}=\mathrm{H}), \mathrm{ABCD} \rightleftarrows \mathrm{BADC})$ and not applicable to the case of nonmutual exchange (5-chlorobenzofuroxan $\left.(\mathrm{R}=\mathrm{Cl}), \mathrm{ABC} \rightleftarrows \mathrm{A}^{\prime} \mathrm{B}^{\prime} \mathrm{C}^{\prime}\right)$. Johnson proposed an elegant modification of the density matrix approach which made it applicable to intramolecular nonmutual exchanges, and the theory was developed by Binsch. ${ }^{8)}$

In the present work, the density matrix method developed by Johnson was applied to the spectral analyses of 5 -fluorobenzofuroxan $(R=F)$, for intramolecular nonmutual exchange between 5-fluorobenzofuroxan $(\mathrm{ABCX})$ and 6-fluorobenzofuroxan $\left(\mathrm{A}^{\prime} \mathrm{B}^{\prime} \mathrm{C}^{\prime} \mathrm{X}^{\prime}\right)$, and the thermodynamic parameters were estimated from the theory of the absolute reaction rate.

\section{Experimental}

Benzofuroxans ${ }^{9}$ (I) are prepared most conveniently by hypochlorite oxidation of 0 -nitroanilines (II) and also by thermal decomposition of o-nitrophenylazides (IV) (Chart 2).

Preparation of 5-Fluorobenzofuroxan by Hypochlorite Oxidation of II-4-Fluoro-2-nitroaniline $\left.{ }^{10}\right)(5 \mathrm{~g})$ was dissolved in a solution of $\mathrm{KOH}(21 \mathrm{~g})$ in $95 \% \mathrm{EtOH}(250 \mathrm{ml})$, producing a moderately red color. The solution was cooled in an ice bath and a $10 \% \mathrm{NaClO}$ solution was added dropwise, with vigorous stirring,

I. Hypochlorite oxidation of 0 -nitroanilines

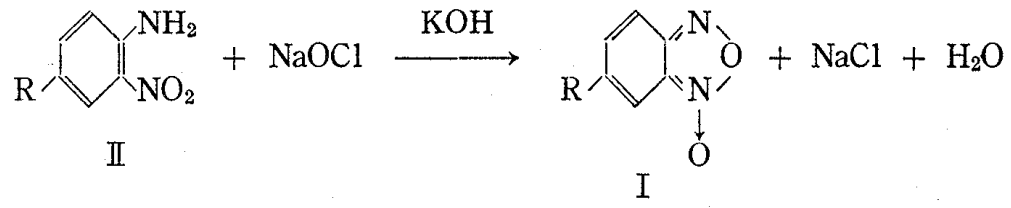

II. Decomposition of o-nitrophenylazide
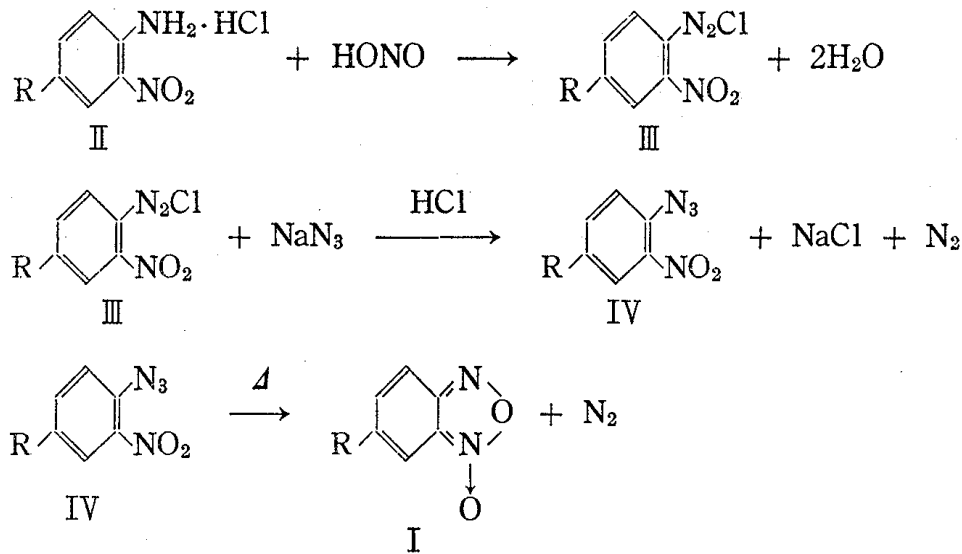

Chart 2

6) J. Karplan, J. Chem. Phys., 28, 273 (1958).

7) S. Alexander, J. Chem. Phys., 37, 967, 974 (1962).

8) C.S. Johnson, Jr., J. Chem. Phys., 41, 3277 (1964); C.S. Johnson. Jr., "Advances in Magnetic Resonance," Vol. 1, ed by J.S. Waugh, Academic Press, Inc., New York, 1965, p. 1; G. Binsch, J. Am. Chem. Soc., 91, 1304 (1969); G. Binsch, “Dynamic Nuclear Magnetic Resonance Spectroscopy," ed. by L.M. Jackman and F.A. Cotton, Academic Press, Inc., New York, 1975, p. 59; H. Nakanishi, Kagaku-no-Ryoiki, 31, 374 (1977).

9) P.A.S. Smith and J.H. Boyer, Org. Syn., 31, 14 (1951).

10) K. Takatori, Y. Yamada, T. Aria, and R. Nakazawa, Yakugaku Zasshi, 78, 108 (1958). 
until the red color disappeared completely. The fluffy yellow precipitate that formed was collected by suctional filtration, washed with $\mathrm{H}_{2} \mathrm{O}$, air-dried, and recrystallized several times from hexane, yielding $1.9 \mathrm{~g}$ $(39 \%)$ of light yellow crystals, mp $48-49^{\circ}$. Anal. Calcd. for $\mathrm{C}_{6} \mathrm{H}_{3} \mathrm{FN}_{2} \mathrm{O}_{2}: \mathrm{C}, 46.76 ; \mathrm{H}, 1.96 ; \mathrm{N}, 18.18$. Found: C, $46.93 ; \mathrm{H}, 2.03 ; \mathrm{N}, 18.28$.

Preparation of 5-Fluorobenzofuroxan by Thermal Decomposition of IV-4-Fluoro-2-nitroaniline (2 g) was dissolved by warming in a suitable amount of $8 \mathrm{~N} \mathrm{HCl}$, the mixture was cooled in an ice-salt bath to keep the temperature between $0^{\circ}$ and $5^{\circ}$ during the addition of a solution of $\mathrm{NaNO}_{2}(2 \mathrm{~g})$ in $10 \mathrm{ml}$ of $\mathrm{H}_{2} \mathrm{O}$. The excess $\mathrm{HNO}_{2}$ was decomposed by addition of urea and the yellow green solution was filtered from a trace of insoluble impurities. The addition of a solution of $\mathrm{NaN}_{3}(2 \mathrm{~g})$ in $10 \mathrm{ml}$ of $\mathrm{H}_{2} \mathrm{O}$ with vigorous stirring gave a. light yellow fluffy precipitate. The precipitate was collected by filtration and, while still wet, was heated at $130^{\circ}$ on an oil bath until evolution of $\mathrm{N}_{2}$ ceased. The resulting material was recrystallized from hexane, yielding $1.2 \mathrm{~g}(58 \%)$ of yellow crystals, $\mathrm{mp} 48-49^{\circ}$. Anal Calcd. for $\mathrm{C}_{6} \mathrm{H}_{3} \mathrm{FN}_{2} \mathrm{O}_{2}: \mathrm{C}, 46.76 ; \mathrm{H}, 1.96 ; \mathrm{N}, 18.18$. Found: $\mathrm{C}, 46.87 ; \mathrm{H}, 1.92 ; \mathrm{N}, 18.28$.

The mixed examination showed that the hypochlorite oxidation product was identical to the thermak decomposition product.

Preparation of NMR Sample___ The compounds were sublimed in vacuum and used as samples for NMR spectral measurement. A solution of $100 \mathrm{mg}$ of 5 -fluorobenzofuroxan in $0.5 \mathrm{ml}$ of a mixed solvent $\left(\mathrm{CDCl}_{3}\right.$ : acetone $=1: 3$ ) was degassed in NMR sample tube under vacuum at $10^{-5}$ Torr by repeated freezing and thawing, then the tube was sealed.

Recording of NMR Spectra_- The NMR spectra were obtained with JNM C60-H and JNM MH-100 spectrometers (Japan Electron Optics Laboratory, Tokyo) equipped with JES-VT-3 variable temperature probe and temperature control, operating at 60 and $100 \mathrm{MHz}$.

The temperature of the solution was calibrated by the use of an iron-constantan thermocouple placed inside a dummy tube and the sample temperature was interpolated with an error of $\pm 1^{\circ}$. The spectra were recorded in the field-frequency sweep mode, with a sweep rate of $0.108 \mathrm{~Hz} / \mathrm{sec}$, and calibration was performed by the usual modulation side-band technique.

\section{NMR Spectra and Spectral Analysis}

\section{Method of Spectral Analysis}

Spectral simulation was performed by the density matrix method ${ }^{8)}$ (case I) and by Pople's treatment ${ }^{11)}$ (case II). The density matrix method was used to simulate all spectra. In addition, Pople's treatment was used for simulation, when chemical exchange relaxation was negligible. Description of these method is given below.

\section{Density Matrix Treatment of Intramolecular Nonmutual Exchange ${ }^{8)}$ (Case I)}

The line shape in the exchange between $A M X$ and $A^{\prime} M^{\prime} X^{\prime}$ spin system can be calculated by the modified Bloch method, $\left.{ }^{12}\right)$ but this method is not applicable to the spin exchange between $\mathrm{ABC}$ and $\mathrm{A}^{\prime} \mathrm{B}^{\prime} \mathrm{C}^{\prime}$ system because the off-diagonal matrix element of the spin Hamiltonian is not negligible in these spin systems. Then the spectra must be analyzed by the density matrix method developed by Johnson, which is a generalization of McConnell's. equations for an intramolecular exchange reaction.

The equation of motion for the density matrix is

$$
\frac{d \boldsymbol{\rho}^{i}}{d t}=-i \boldsymbol{L}^{i} \cdot \boldsymbol{\rho}^{i}+\sum_{j=1}^{N} \boldsymbol{\rho}^{j} \cdot \boldsymbol{D}_{j i}\left(P_{j} / P_{i}\right)
$$

where $\boldsymbol{\rho}^{i}$ is the density matrix vector of the $i$-th spin configuration, $\boldsymbol{L}^{i}$ is the Liouville operator associated with the $i$-th Hamiltonian in the absence of the spin exchange, and $\boldsymbol{D}$ is the transition-probability matrix defined as $\boldsymbol{D}_{i i}=-1 / \tau_{i}$, and $\boldsymbol{D}_{i j}=p_{j i} / \tau_{j}(i \neq j)$, where $\tau_{j}$ is the mean life time of the $j$-th spin configuration. The fractional population of the $j$-th spin configuration is $P_{j}$; when a transition away from the $j$-th spin configuration occurrs, $p_{i j}$ gives the transition probability jumping from the $j$-th to $i$-th spin configuration.

11) J.A. Pople, Mol. Phys., 1, 168 (1958); J.M. Anderson and J.D. Baldeschwieler, J. Chem. Phys., 40, 3241 (1964).

12) H.M. McConnell, J. Chem. Phys., 28, 430 (1958). 
As an example of the use of Eq.(1), consider the intramolecular nonmutual exchange between 5-halobenzofuroxan (spin configuration A) and 6-halobenzofuroxan (B) in Chart 1 . This PMR spectrum is analyzed as an exchange $A B C \rightleftarrows A^{\prime} B^{\prime} C^{\prime}$. If $\tau_{\mathrm{A}}^{-1}$ is the rate of the change $\mathrm{A} \rightarrow \mathrm{B}$, and $\tau_{\mathrm{B}}{ }^{-1}$ that for $\mathrm{B} \rightarrow \mathrm{A}$, a steady state is reached when

$$
P_{\mathrm{B}} / \tau_{\mathrm{B}}=P_{\mathrm{A}} / \tau_{\mathrm{A}}, \quad P_{\mathrm{A}}+P_{\mathrm{B}}=1 \text {. }
$$

Thus the equations of motion of $\boldsymbol{\rho}_{\mathrm{A}}$ and $\boldsymbol{\rho}_{\mathrm{B}}$ for the transition between the state $i$ and $j$ become respectively

and

$$
\frac{d \boldsymbol{\rho}_{i j}^{\mathrm{A}}}{d t}=-i\left[\mathscr{H}_{\mathrm{A}} \cdot \boldsymbol{\rho}_{\mathrm{A}}\right]_{i j}-\frac{\boldsymbol{\rho}_{i j}^{\mathrm{A}}}{T_{2 \mathrm{~A}}}-\frac{\boldsymbol{\rho}_{i j}^{\mathrm{A}}}{\tau_{\mathrm{A}}}+\frac{\boldsymbol{\rho}_{i j}^{\mathrm{B}}}{\tau_{\mathrm{A}}}
$$

$$
\frac{d \boldsymbol{\rho}_{i j}^{\mathrm{B}}}{d t}=-i\left[\mathscr{H}_{\mathrm{B}} \cdot \rho_{\mathrm{B}}\right]_{i j}-\frac{\boldsymbol{\rho}_{i j}^{\mathrm{B}}}{T_{2 \mathrm{~B}}}-\frac{\boldsymbol{\rho}_{i j}^{\mathrm{B}}}{\tau_{\mathrm{B}}}+\frac{\boldsymbol{\rho}_{i j}^{\mathrm{A}}}{\tau_{\mathrm{B}}},
$$

where the line width due to spin relaxation processes is accounted for by adding a term $1 / T_{2}$ to Eq.(1), and $T_{2}$ is the spin-spin relaxation time. The Hamiltonian for configuration $\mathrm{A}$ can be written as

$$
\mathscr{H}_{\mathrm{A}}=\sum_{i} I_{\mathrm{ZA}}^{i}\left(\omega_{i}-\omega\right)+2 \pi \sum_{i<j} J_{\mathrm{Aj}}^{\mathrm{A}} \cdot \boldsymbol{I}_{\mathrm{A}}^{i} \cdot \boldsymbol{I}_{\mathrm{A}}^{j}+\sum_{i} \gamma^{i} \cdot \boldsymbol{H}_{1} \cdot I_{\mathrm{XA}}^{i},
$$

and similarly for B. For slow passage experiments without saturation, all of the time derivatives in Eq.(3) and (4) are equal to zero, and only the transition under the condition $A M=1$ is considered. Then Eq.(3) and (4) would lead to 30 linear equations in the case of threespin systems. The intensity of absorption, $I(\omega)$, is proportional to the expectation value of $I_{\mathrm{y}}(y$-axis component of spin operator $\mathbf{I})$ in the rotating frame,

$$
I(\omega)=\operatorname{kIm}\left\{P_{\mathrm{A}} \cdot \operatorname{Tr}\left(\rho_{\mathrm{A}} \cdot \sum_{j} I_{+\mathrm{A}}^{j}\right)+P_{\mathrm{B}} \cdot \operatorname{Tr}\left(\rho_{\mathrm{B}} \cdot \sum_{j} I_{+\mathrm{B}}^{j}\right)\right\},
$$

where $k$ is a proportionality factor, $T r$ the trace, $I m$ the imaginary part, and $I_{+\mathrm{A}}^{\mathrm{i}}$ the raising operator $\left(I_{x}+i I_{y}\right)$ for the $j$-th spin in the configuration A. Although the form of both matrices $I+$ and $\rho$ depends on choice of the basis set, the trace is independent of the choice. For the sake of convenience, we have chosen a representation where the basis set are basic product spin functions. These processes were programmed in FORTRAN IV, and the computation was carried out with a digital computer, NEAC 2200-375 (Nippon Electric Co., Tokyo). For a given set of $\tau_{\mathrm{A}}, P_{\mathrm{A}}, T_{2}$, chemical shifts, and spin-spin coupling constants, the theoretical spectra were calculated by the computer, and the calculated values were plotted by hand to obtain the line shapes. For a spectrum of about 400 points, ca. 30 min of computation time was required.

\section{Treatment by Pople's Method (Case II)}

This method was applied to the analysis of spectra obtained at low temperatures.

As a general rule, the NMR spectra were analyzed by using the iteration of the least squares method, ${ }^{13)}$ yielding values of the chemical shifts, coupling constants, and line spectra. In the present work, the relative line width was calculated using Pople's treatment ${ }^{11)}$ as shown in the following. Calculation of the line width is accomplished by the following assumption; i.e., a relaxation mechanism is dominant, the line widths are small compared with separation between lines of a given multiplet, and the relaxation process is a Markovian random process. From these assumptions the width of a line is simply given as

$$
W_{m-m^{\prime}}=\frac{1}{2}\left(\sum_{n \neq m} P_{m-n}+\sum_{n \neq m^{\prime}} P_{m^{\prime}-n}\right)
$$

where $W_{m-m^{\prime}}$ is the width of the spectral line resulting from a transition $m \rightarrow m^{\prime}$, and $P_{m-n}$ is the transition probability from state $m$ to state $n . \quad P_{m-m^{\prime}}$ is proportional to

13) S. Castellano and A.A. Bothner-By, J. Chem. Phys., 41, 3863 (1964). 
$\left\langle\left. i\left\langle m\left|\mathscr{H}^{\prime}\right| m^{\prime}\right\rangle\right|^{2}\right\rangle_{\mathrm{Av}}$, where $\mathscr{H}^{\prime}$ is the relaxation operator and formulated by the isotropic random field relaxation mechanism. The spectral contours were calculated according to

$$
I(\nu)=\sum L\left(\nu, \nu_{i}, W_{i}\right) \cdot S_{i},
$$

where $I(v)$ is the intensity at a frequency $v, L\left(v, v_{i}, W_{i}\right)$ is the Lorentzian shape function for frequency $v$ and a line $i$ at frequency $v_{i}$ width $W_{i}$, and relative intensity $S_{i}$.

\section{NMR Spectra}

The $100 \mathrm{MHz}$ spectrum of 5 -fluorobenzofuroxan at $-43^{\circ}$ is shown by the superposition of the spectra of 5-isomer and 6-isomer, both of which are found in the typical patterns of the $\mathrm{ABC}$ part of the $\mathrm{ABCX}$ spin system, $\mathrm{X}$ being the ${ }^{19} \mathrm{~F}$ nucleus. The spectrum indicates a fairly slow rearrangement, and the fractional population of 5 -isomer is 0.71 and the freeenergy difference between the tautomers, $\Delta F$, is about $400 \mathrm{cal} / \mathrm{mol}$.

a
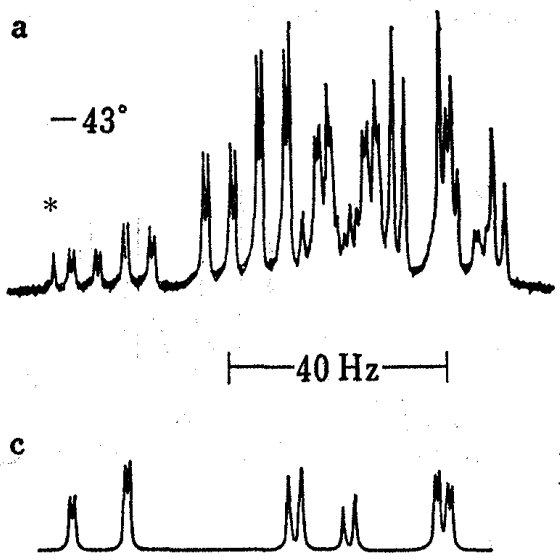

e

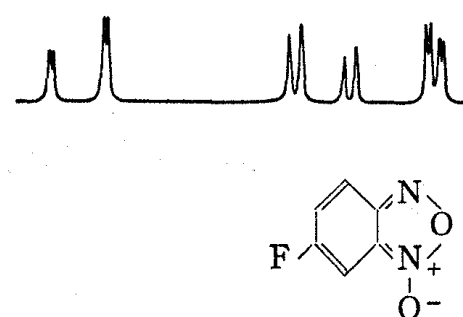

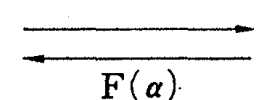
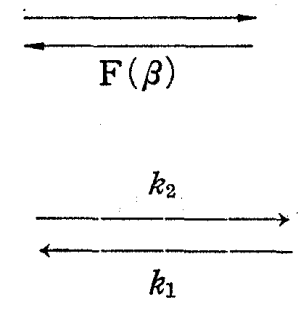

b

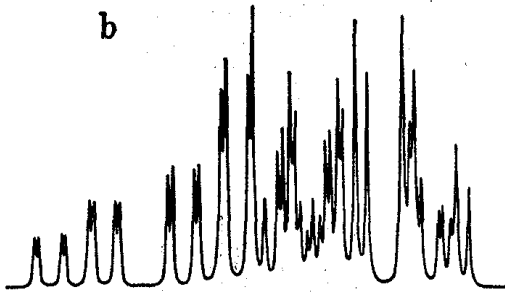

d

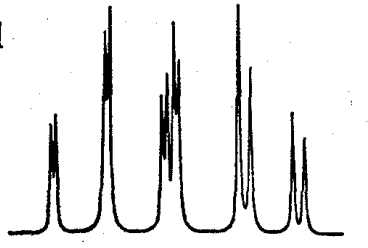

f

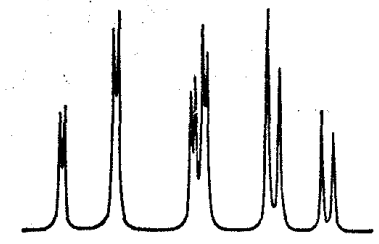

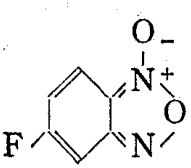

Fig. 1. Experimental and Theoretical Spectra for 5- and 6-Fluorobenzofuroxan

a) PMR spectrum at $100 \mathrm{MHz}$ and $-43^{\circ}$; b) theoretical spectrum obtained by Pople's model assuming isotropic random field relaxation, superposition of $c, d, e$ and $f ; c)$ calculated line shape of 6-fluorobenzofuroxan with $\alpha$-spin of ${ }^{19} \mathrm{~F}$ nucleus; $d$ ) calculated line shape of 5 -fluorobenzofuroxan with $\alpha$-spin of ${ }^{10} \mathrm{~F}$ nucleus; $e$ ) calculated line shape of 6 -isomer with $\beta$-spin of ${ }^{19} \mathrm{~F}$; $d$ ) calculated line shape of 5 -isomer with $\beta$-spin of ${ }^{10} \mathrm{~F}$.

*) singlet caused by chloroform in deuterochloroform.

TaBLE I. Coupling Constants $(\mathrm{Hz})$

\begin{tabular}{llllllllllll}
\hline & \\
\hline
\end{tabular}


TABLE II. Chemical Shifts ${ }^{a}$ )

\begin{tabular}{|c|c|c|c|c|}
\hline \multirow{2}{*}{$\begin{array}{l}\text { Substituted } \\
\text { benzofuroxan }\end{array}$} & \multicolumn{4}{|c|}{ Chemical shift $(\mathrm{Hz})$ of proton } \\
\hline & $v_{4}$ & $\nu_{5}$ & $\nu_{6}$ & $\nu_{7}$ \\
\hline 5-Fluoro-(60 MHz) & 318.50 & - & 308.30 & 328.90 \\
\hline 6-Fluoro-(60 MHz) & 343.28 & 318.90 & - & 306.56 \\
\hline 5 -Fluoro- $(100 \mathrm{MHz})^{*}$ & 21.30 & - & 3.57 & 39.02 \\
\hline 6-Fluoro-(100 MHz)* & 63.81 & 21.86 & - & 0.00 \\
\hline
\end{tabular}

a) Chemical shifts were measured from acetone signal, and the astarisk marks indicate the values from $\mathrm{H}-7$ proton chemical shift of 6 -fluorobenzofuroxan.

It was assumed that 5-fluorobenzoxan is more stable than 6-fluorobenzofuroxan, according to the $\mathrm{NMR}^{4)}$ and $\mathrm{X}$-ray ${ }^{14)}$ data of 5-chlorobenzofuroxan, namely, crystals of 5-chlorobenzofuroxan consist of only the 5-tautomer, and not the 6 -tautomer or mixtures of 5 - and 6-tautomer. Application of Pople's method (case II) gave the theoretical spectrum and NMR parameters as shown in Fig. 1 and Table I and II.
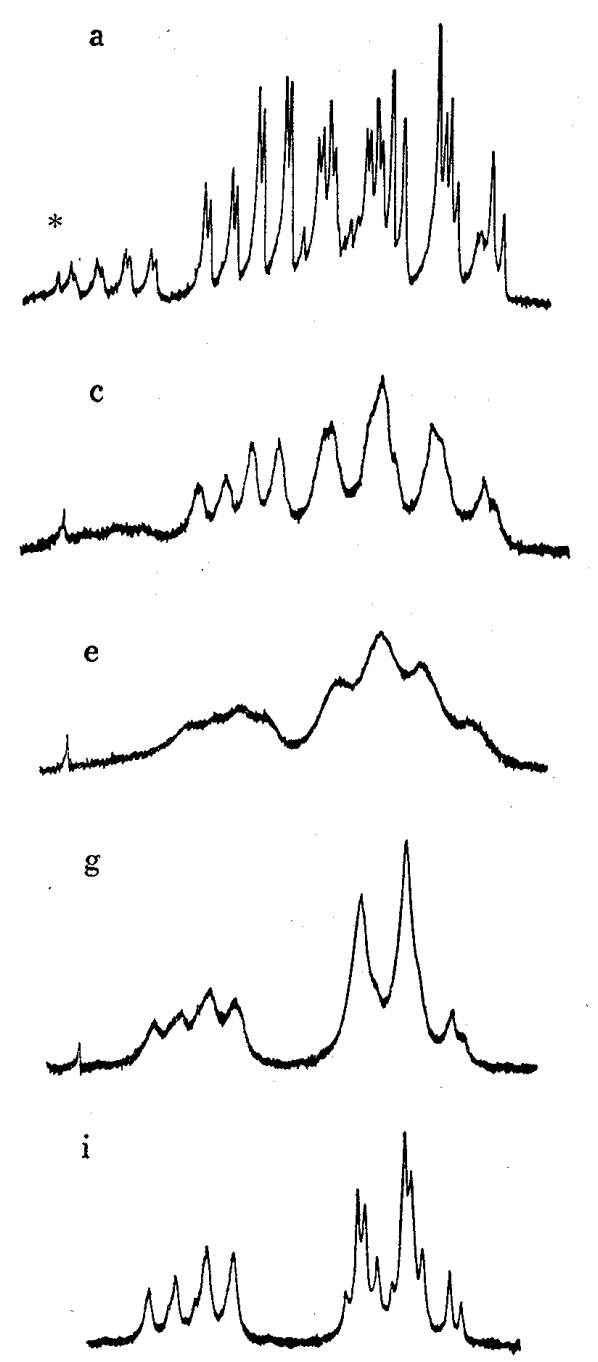

Fig. 2. Experimental and Theoretical Spectra for Intramolecular Rearrangement between 5- and 6-Fluorobenzofuroxan at $100 \mathrm{MHz}$

The theoretical spectra are calculated by means of the density matrix method.

*) signal of chloroform.

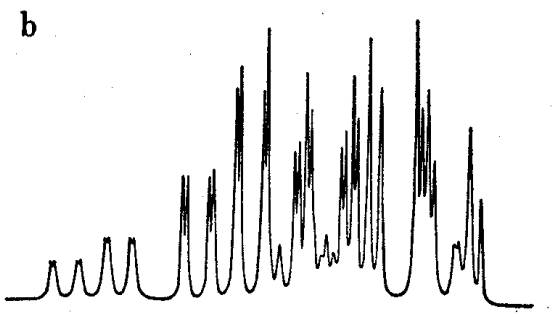

$d$

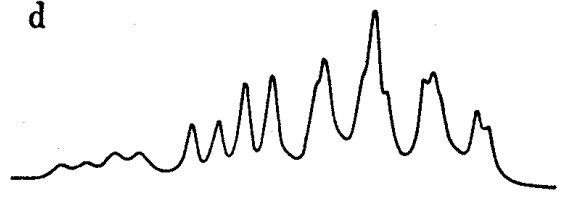

$$
\begin{aligned}
\tau & =0.06 \\
t & =0.0^{\circ}
\end{aligned}
$$

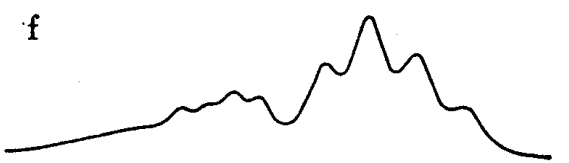

h

$$
\begin{aligned}
\tau & =0.006 \\
t & =23^{\circ}
\end{aligned}
$$$$
\tau=0.0015
$$$$
t=35^{\circ}
$$

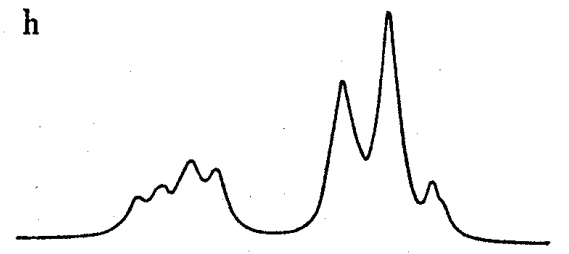

j

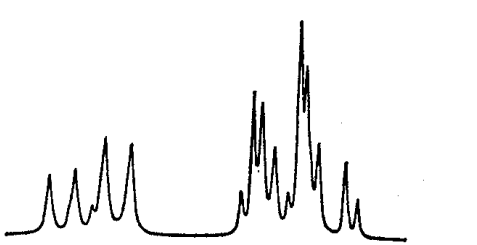

14) D. Britton and W.E. Noland, J. Org. Chem., 27, 3218 (1962). 
In particular, this spectral analysis was simplified according to the following the technique of subspectral analysis. ${ }^{15)}$ The $\mathrm{ABC}$ part of the $\mathrm{ABCX}$ spectrum can be broken down into two abc subspectra, which may be denoted as $a^{+} b^{+} c^{+}$and $a^{-} b^{-} c^{-}$, respectively, where the effective chemical shifts, $\nu_{\mathrm{a}}{ }^{+}$etc., are determined from the following equations.

$$
\begin{aligned}
& \nu_{\mathrm{a}}^{ \pm}=\nu_{\mathrm{A}} \pm \frac{1}{2} J_{\mathrm{AX}}, \\
& \nu_{\mathrm{b}}^{ \pm}=\nu_{\mathrm{B}} \pm \frac{1}{2} J_{\mathrm{BX}}, \\
& \nu_{\mathrm{c}}^{ \pm}=\nu_{\mathrm{C}} \pm \frac{1}{2} J_{\mathrm{cX}} .
\end{aligned}
$$

The abc spectra of the 5-isomer in Fig. 1-d,-f and that of the 6-isomer in Fig. 1-c,-e can be picked out by means of an $\mathrm{ABC}$ analysis. The technique of subspectral analysis was also used to calculate the theoretical spectra by the density matrix method. Providing that the chemical exchange does not affect the ${ }^{19} \mathrm{~F}$ nuclear spin inversion, the spectra due to an exchange between $\mathrm{ABCX}$ and $\mathrm{A}^{\prime} \mathrm{B}^{\prime} \mathrm{C}^{\prime} \mathrm{X}^{\prime}$ can be analyzed as an exchange between $\mathrm{a}^{+} \mathrm{b}^{+} \mathrm{c}^{+}$and $a^{\prime}+b^{\prime}+c^{\prime+}$, and between $a^{-} b^{-} c^{-}$and $a^{\prime}-b^{\prime}-c^{\prime}-$. Namely, these may be shown by the following rate equations:
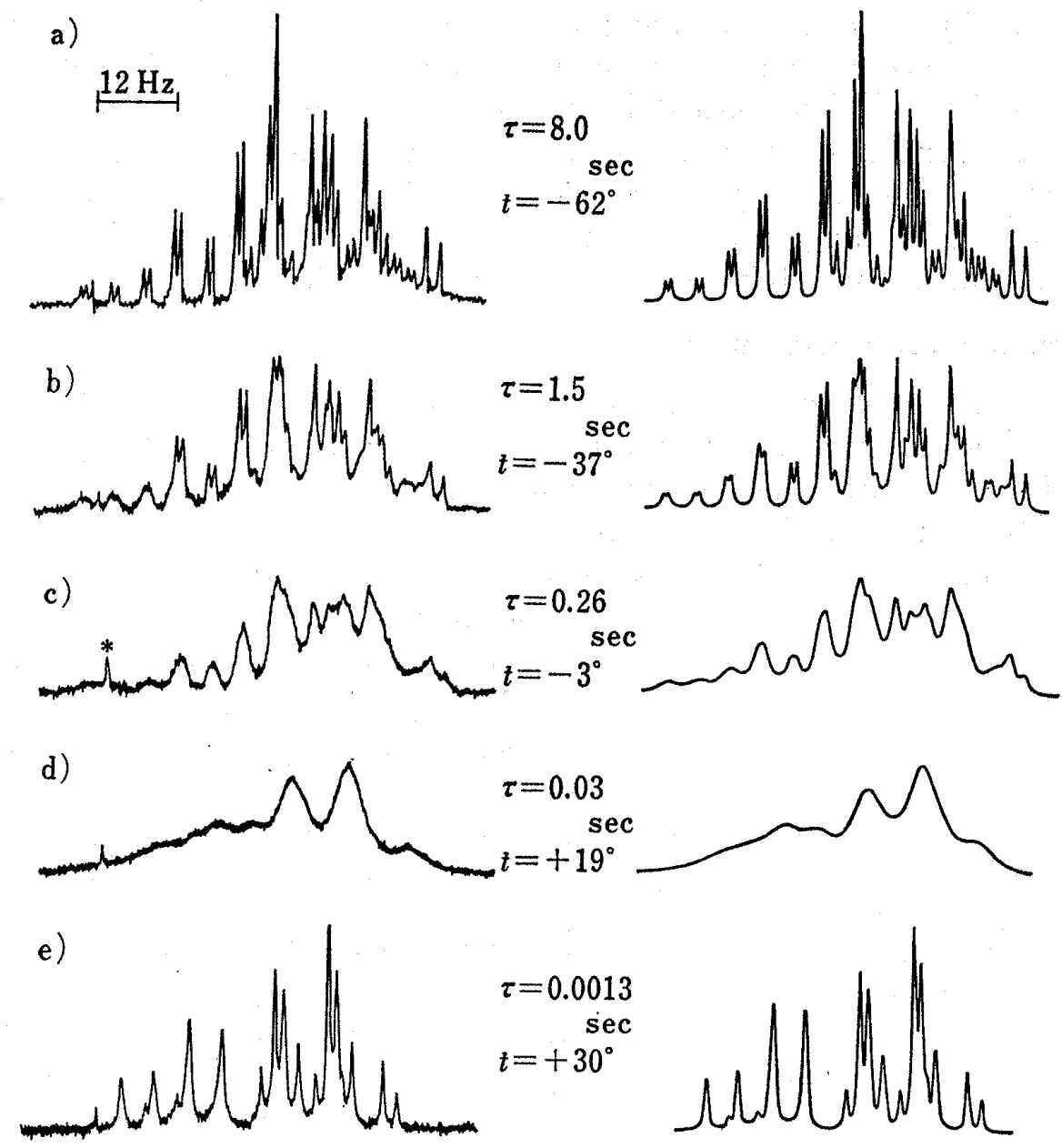

Fig.3. Experimental and Theoretical Spectra for Intramolecular Rearrangement between 5- and 6-Fluorobenzofuroxan at $60 \mathrm{MHz}$

The theoretical spectra are calculated by means of the density matrix method. *) signal of chloroform.

15) P. Diehl, R.K. Harris, and R.G. Jones, "Progress in Nuclear Magnetic Resonance Spectroscopy," Vol. 3, Pergamon Press, Oxford, 1967, p. 1. 


$$
\begin{aligned}
& \mathrm{F}(\alpha) \text {-5-isomer } \underset{k_{2}}{\stackrel{k_{1}}{\rightleftharpoons}} \mathrm{F}(\alpha) \text {-6-isomer } \\
& \mathrm{F}(\beta)-5 \text {-isomer } \underset{k_{2}}{\stackrel{k_{1}}{\rightleftharpoons}} \mathrm{F}(\beta)-6 \text {-isomer }
\end{aligned}
$$

where $F(\alpha)$-5-isomer denotes 5 -isomer possessing $\alpha$-spin of ${ }^{19} \mathrm{~F}$ nucleus, and similarly for the other. By this technique, the $112 \times 122$ coefficient matrix of corresponding density matrix decomposed into two $30 \times 30$ matrices and the computation time was largely reduced. Experimental and theoretical spectra are shown in Fig. 2. Similarly the results of the spectral analyses at $60 \mathrm{MHz}$ are shown in Fig. 3 and in Table I and II.

\section{Results and Discussion}

\section{Problem of Spectral Simulation}

In general, good agreement is found between theoretical and experimental spectra, and the experimental spectrum at a low temperature shows better agreement with the spectrum calculated by Pople's method rather than by the density matrix method. Accuracy of the life time obtained by the density matrix method depends on the chemical shifts because the temperature dependence of spin-spin coupling constants is less than that of chemical shifts, namely, the problem of this method is that chemical shifts cannot be determined with accuracy at all temperatures. In this study, the spectral simulation is carried out as chemical shifts are corrected at each temperature. The chemical shifts were found to vary slightly with temperature in the range from $-43^{\circ}$ to $+30^{\circ}$, and an error of a few hertz at each temperature had a comparatively little effect on the evaluated life time.

\section{Problem of Temperature Measurements}

It can be seen from Table III that the difference between the parameters at 60 and 100 $\mathrm{MHz}$ is greater than the random error of the parameters; the differences are attributable to uncertainty of temperature measurements rather that of temperature controls. It can therefore be presumed that the NMR sample temperature on-spinner differs from off-spinner and the temperature difference at $60 \mathrm{MHz}$ is greater than that at $100 \mathrm{MHz}$; as a result, our tem-

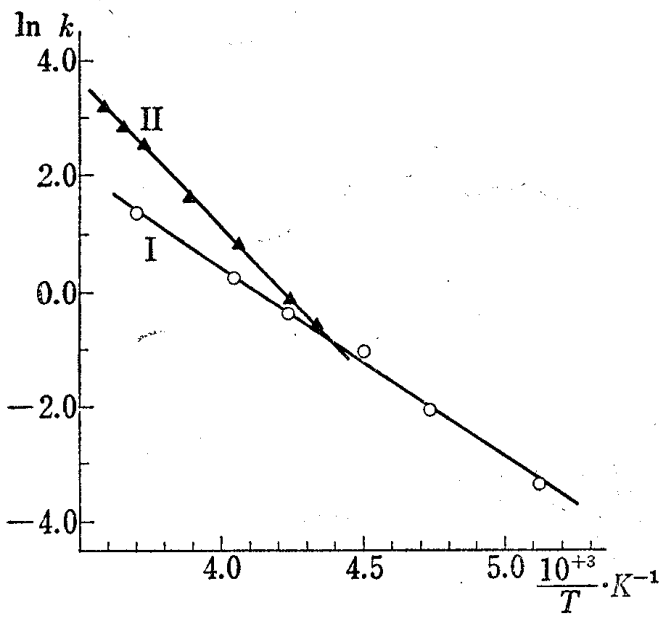

Fig. 4. Arrhenius Plots of the Kinetic Data for 5-Fluorobenzofuroxan at $60 \mathrm{MHz}$ (I) and $100 \mathrm{MHz}$ (II) perature measurements are not very good. To avoid this problem in the study of 2-acetylfuran, Dahlquist and Forsén ${ }^{16)}$ performed temperature measurements by the use of temperature dependence of the shift difference in $\mathrm{OH}$ and $\mathrm{CH}_{3}$ methanol signals, and showed that the temperature measurements are reliable.

\section{Intramolecular Rearrangement Reaction}

The experimental rate data have been analyzed as follows: the Arrhenius energy of activation, $E_{\mathrm{a}}$, and the frequency factor, $A$, are calculated in the normal way by means of the equation $k=1 / \tau=A \cdot \exp \left(-E_{\mathrm{a}} / \boldsymbol{R} T\right)$ from leastsquares fit of $\ln (k)$ vs. $1 / T$, as shown in Fig. 4. The free energy of activation, $\Delta F^{\star}$, enthalpy of activation, $\Delta H^{\ddagger}$, and entropy of activation,

16) K-I. Dahlqvist and S. Forsén, Acta. Chem. Scand., 24, 672 (1970). 
TABLE III. Thermodynamic Quantities for the Intramolecular Rearrangement between 5- and 6-Fluorobenzofuroxan $a$ )

\begin{tabular}{lrcccc}
\hline \hline $\begin{array}{l}\text { Substituted } \\
\text { benzofuroxan }\end{array}$ & $\begin{array}{c}E_{\mathrm{a}} \\
\mathrm{kcal} / \mathrm{mol}\end{array}$ & $\log A$ & $\begin{array}{c}\Delta H^{*} \\
\mathrm{kcal} / \mathrm{mol}\end{array}$ & $\begin{array}{c}\Delta S^{*} \mathrm{cal} / \mathrm{deg} \cdot \mathrm{mol} \\
\mathrm{cal} / \mathrm{mol}\end{array}$ \\
\hline 5-Fluoro-(60 MHz) & $6.55 \pm 0.09$ & $5.89 \pm 0.09$ & $6.10 \pm 0.09$ & $-33.0 \pm 0.4$ & 410 \\
6-Fluoro-(60 MHz) & $6.14 \pm 0.09$ & $5.89 \pm 0.09$ & $5.69 \pm 0.09$ & $-33.0 \pm 0.4$ & \\
5-Fluoro-(100 MHz) & $10.21 \pm 0.13$ & $9.40 \pm 0.12$ & $9.70 \pm 0.13$ & $-17.2 \pm 0.5$ & 410 \\
6-Fluoro-(100 MHz) & $9.79 \pm 0.13$ & $9.40 \pm 0.12$ & $9.29 \pm 0.13$ & $-17.2 \pm 0.5$ & \\
\hline
\end{tabular}

a) The errors are average deviations (assuming only random errors).

$\Delta S^{\ddagger}$, in the theory of the absolute reaction rate are calculated directly from the fundamental rate Eq. (11). ${ }^{17}$

$$
k=1 / \tau=\frac{k T}{h} \exp \left(-\Delta F^{\ddagger} / R T\right)=\frac{k T}{h} \exp \left(\Delta S^{\ddagger} / R\right) \exp \left(-\Delta H^{\ddagger} / R T\right)
$$

The activation parameters are summarized in Table III. From the above argument, the following results are derived.

(1) 5-Fluorobenzofuroxan is more stable than 6-fluorobenzofuroxan and $\Delta F$ is ca. 400 $\mathrm{cal} / \mathrm{mol}$.

(2) The intramolecular rearrangement is the first-order reaction via a transition state.

(3) The activation parameters are found to be $\Delta H^{*}=5 \sim 10 \mathrm{kcal} / \mathrm{mol}$ and $\Delta S^{*}=-33$ $\sim-17 \mathrm{cal} / \mathrm{deg} . \mathrm{mol}$.

The method of analyses are applied for a series of 5-halobenzofuroxan and the mechanism of the exchange reaction are interpreted. The kinetic results, together with some energy calculation, will be given in a subsequent paper.

Acknowledgement The authors are indebted to the Computer Center of Shizuoka Prefectural Government for the use of their computer for the present work, and to Asahi Chemical Industry Co., Shizuoka, and to Dr. K. Taniguchi of this Company for the use of their NMR spectrometer.

17) S. Glasston, K.J. Laidler and H. Eyring, "The Theory of Rate Process," MaGraw-Hill Book Co., Inc., New York, 1941. 